\title{
Calculation of the Periodic Spectral Components in a Chaotic DC-DC Converter
}

\author{
Jonathan H. B. Deane, Peter Ashwin, David C. Hamill, Senior Member, IEEE, and David J. Jefferies
}

\begin{abstract}
A simple mapping is derived, which describes the behavior of a peak current-mode controlled boost converter operating chaotically. The invariant density of this mapping is calculated iteratively and, from this, the power density spectrum of the input current at the clock frequency and its harmonics is deduced. The calculation is presented, along with experimental verification. The possibility of a novel application of chaos-amelioration of power supply interference-is discussed.
\end{abstract}

\section{INTRODUCTION}

I $\mathrm{N}$ a previous publication [1] we presented the idea that chaos, a naturally occurring phenomenon in switch-mode power supplies, might be used to improve their electromagnetic compatibility (EMC). We illustrated this proposal with some measurements. In the present paper we show how to calculate the power density spectrum (PDS), at the clock frequency and its harmonics, of the input current of a currentmode controlled boost converter operating chaotically. This work builds mainly on [2]-[4], in which we derived an exact two-dimensional (2-D) mapping in closed form that describes the behavior of this converter. The requirement for low output voltage ripple in practical designs leads to a circuit that is well described by a simpler one-dimensional (1-D) mapping, derived in this paper. We calculate its invariant density and, from this, the periodic components in the PDS of the inductor current are found. We then confirm experimentally and numerically the validity of the model and our PDS calculation.

We also discuss briefly the implications for EMC improvement by chaos. The electromagnetic interference requirements for switch-mode power supplies are, in essence, that the input current PDS should be less than a specified frequencydependent level. Hence, our demonstration that chaos can be exploited to reduce spectral peaks, significantly in some cases, leads us to propose EMC compliance by chaos.

\section{THE SIMPLIFIED MAPPING}

A 2-D mapping that describes the boost converter in Fig. 1 was presented in [2]. Another 2-D version, taking into account inductor and capacitor parasitics, appears in [5].

Manuscript received March 11, 1998; revised August 18, 1998 and October 27, 1998. The work of P. Ashwin was supported in part by a Newly Appointed Science Lecturer Grant from the Nuffield Foundation. This paper was recommended by Associate Editor H. Kawakami.

The authors are with the School of Electronic Engineering, Information Technology and Mathematics, University of Surrey, Guildford GU2 5XH, U.K.

Publisher Item Identifier S 1057-7122(99)09263-6.

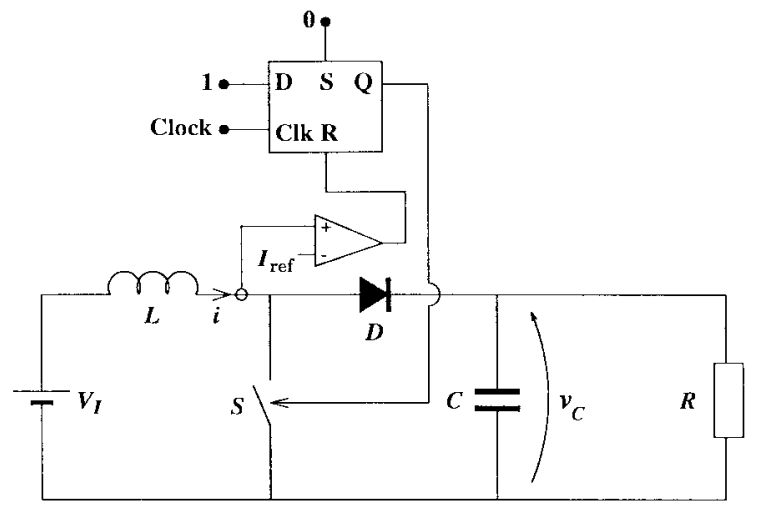

Fig. 1. The peak current-mode controlled boost converter.

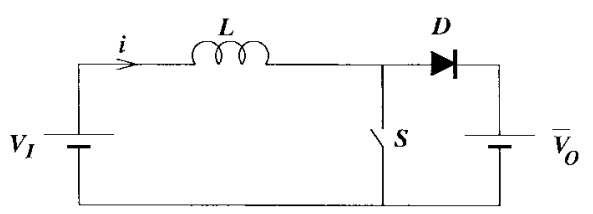

Fig. 2. Simplified model of the power circuit of the boost converter.

The mapping that describes a boost converter as it is usually designed, however, can be simplified to a 1-D version that, nonetheless, captures the features of interest in the behavior of the real circuit. In order to derive this 1-D mapping, we make the following assumptions.

1) The capacitor voltage $v_{c}$ is sufficiently close to a constant $\bar{V}_{O}$ that the inductor current waveform can be approximated by a linear ramp at all times. This requires that $C R \gg T_{c}$ where $T_{c}$ is the clock period. In practical circuits, $C$ is sufficiently large for this to be valid.

2) All components are ideal.

3) The clock pulses are of infinitesimal duration and period $T_{c}$.

With these assumptions, the power circuit can be modeled in the simplified form shown in Fig. 2. The switch $S$ is controlled by feedback. It closes when a clock pulse arrives and opens at the instant when $i(t)$ reaches the reference current $I_{\text {ref }}$.

The inductor current $i(t)$ is sketched in Fig. 3. While $S$ is closed, $i(t)$ satisfies

$$
\frac{d i}{d t}=\frac{V_{I}}{L}
$$

If $t=0$ at the instant of $S$ closing, then $i(t)$ is

$$
i(t)=i_{n}+\frac{V_{I}}{L} t
$$




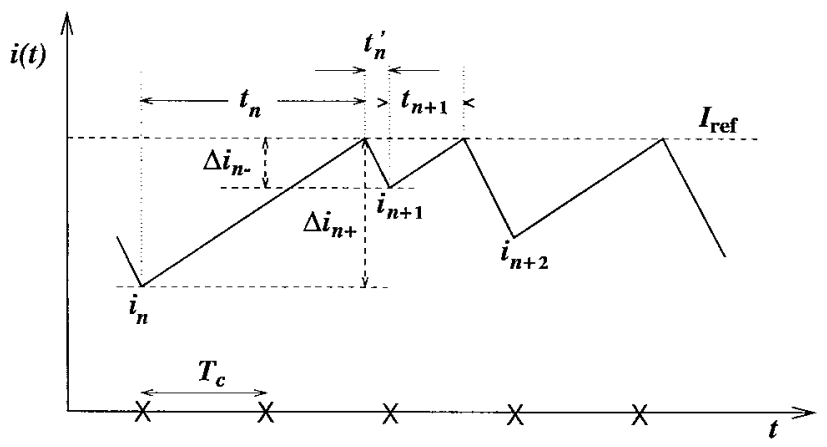

Fig. 3. The current waveform $i(t)$ in the simplified boost converter.

valid until time $t_{n}$ at which $i\left(t_{n}\right)=I_{\text {ref. }}$ Using (1)

$$
t_{n}=\frac{\left(I_{\mathrm{ref}}-i_{n}\right) L}{V_{I}} .
$$

Switch $S$ now opens and $i(t)$ is given by

$$
\frac{d i}{d t}=\frac{V_{I}-\bar{V}_{O}}{L}
$$

with initial condition $i(0)=I_{\text {ref }}$. (We reset the time origin for convenience.) Hence

$$
i(t)=I_{\mathrm{ref}}+\frac{V_{I}-\bar{V}_{O}}{L} t
$$

until the next clock pulse arrives. This happens at a time

$$
t_{n}^{\prime}=T_{c}-T_{c}\left(\frac{t_{n}}{T_{c}}\right) \bmod 1
$$

after $S$ last opened.

The mapping $i_{n} \mapsto i_{n+1}$ can now be derived. By definition, $i_{n+1}=i\left(t_{n}^{\prime}\right)$ which, according to (2)-(4), is

$$
i_{n+1}=I_{\mathrm{ref}}+\frac{\left(V_{I}-\bar{V}_{O}\right) T_{c}}{L}\left[1-\left(\frac{\left(I_{\mathrm{ref}}-i_{n}\right) L}{V_{I} T_{c}}\right) \bmod 1\right] .
$$

Introducing a dimensionless time $x_{n}$, defined as

$$
x_{n}=\frac{t_{n}}{T_{c}}=\frac{\left(I_{\mathrm{ref}}-i_{n}\right) L}{V_{I} T_{c}}
$$

and parameter $\alpha$, defined as

$$
\alpha=\frac{\bar{V}_{O}}{V_{I}}-1
$$

the mapping (5) can be written in the simplified form

$$
x_{n+1}=F\left(x_{n}\right)=\alpha\left(1-x_{n} \bmod 1\right) .
$$

Since $\bar{V}_{O}>V_{I}$ for a boost converter $\alpha>0$.

Note that the switching times can be recovered from (6)

$$
t_{n}=T_{c} x_{n} \quad \text { and } \quad t_{n}^{\prime}=T_{c}\left(1-x_{n} \bmod 1\right) .
$$

When $\alpha>1$, the sequence $\left\{x_{0}, x_{1}, \ldots, x_{n} \ldots\right\}$ will be chaotic with each $x_{n}$ lying between 0 and $\alpha$. The mapping (6), and scaled versions of it, have been studied in several contexts, most notably by Rényi [6] and [7, sec. 6.2]. The fact that $F$ is the Rényi transformation means that the Perron-Frobenius operator $P$, defined in the Appendix, has an invariant density $\rho(x)$ that is 1) absolutely continuous with respect to the Lebesgue measure on the interval $[0, \alpha)$ and 2) ergodic and asymptotically stable [7, Th. 6.2.1]. This density is calculated in the Appendix.

\section{Approximation OF the MeAn State VARIABLES}

In order to use the mapping (6), we need to know the value of $\alpha\left(=\bar{V}_{O} / V_{I}-1\right)$ and, hence, there remains the problem of relating $\bar{V}_{O}$, the mean output voltage, to known quantities in the circuit. We obtain an estimate of $\bar{V}_{O}$ by assuming that $S$ is closed on average for a time $\bar{D} T_{c}$ per clock cycle and $\bar{V}_{O}$ is constant. Here, $\bar{D}$ is to be interpreted as the mean duty factor of $S$. On average

$$
\bar{V}_{O}=R(1-\bar{D})\left(I_{\text {ref }}-\frac{\overline{\Delta i}}{2}\right)
$$

where $R$ is the load resistance shown in Fig. 1. This assumes that the current through the diode is either zero (for a fraction $\bar{D}$ of the time) or $I_{\text {ref }}-\overline{\Delta i} / 2$ (for a fraction $1-\bar{D}$ of the time). When $S$ is closed, $i$ rises at a rate of $V_{I} / L$ for a time $\bar{D} T_{c}$, on average. When $S$ is open, $i$ falls at a rate $\left(\bar{V}_{O}-V_{I}\right) / L$ for a time $(1-\bar{D}) T_{c}$. In terms of the mean current rise $\overline{\Delta i_{+}}$ and fall $\overline{\Delta i}_{-}$

$$
\begin{aligned}
& \overline{\Delta i}_{+}=\frac{V_{I}}{L} \bar{D} T_{c} \quad \overline{\Delta i}_{-}=\frac{\bar{V}_{O}-V_{I}}{L}(1-\bar{D}) T_{c} \quad \text { and } \\
& \overline{\Delta i}_{+}=\overline{\Delta i}_{-}=\overline{\Delta i} .
\end{aligned}
$$

Eliminating $\overline{\Delta i}_{+}, \overline{\Delta i}_{-}, \overline{\Delta i}$ and $\bar{D}$ between (8) and (9) gives

$$
\bar{V}_{O}^{3}+\bar{V}_{O}\left(V_{I} T_{c} / 2 L-I_{\text {ref }}\right) R V_{I}-R T_{c} V_{I}^{3} / 2 L=0
$$

which can be solved for $\bar{V}_{O}$ by selecting the real $\operatorname{root} \bar{V}_{O}>V_{I}$. Hence, $\alpha$ can be found.

\section{The POWER Density SPECTRUM OF THE INDUCTOR CURRENT}

We now calculate the PDS of the current through the inductor $i(t)$ from the invariant density of the mapping $F$. To this end, we first discuss the autocorrelation function of the current $R_{i}(\tau)$ and calculate its Fourier transform under certain assumptions. We then apply Birkhoff's Ergodic Theorem [9] to calculate the periodic component of the PDS and obtain results both for periodic and chaotic operation of the converter.

Recall that the PDS, which we will write as $\tilde{I}(\omega)$, is the generalized Fourier transform of the autocorrelation function

$$
R_{i}(\tau)=\lim _{T \rightarrow \infty} \frac{1}{2 T} \int_{-T}^{T} i(t) i(t+\tau) d t .
$$

Experimental and numerical evidence suggests that we can write the inductor current as

$$
i(t)=p(t)+c(t)
$$

so that the current $i(t)$ splits into a periodic $(p)$ and nonperiodic $(c)$ component. We now extract the periodic component by considering

$$
A(\omega)=\lim _{T \rightarrow \infty} \frac{1}{2 T} \int_{-T}^{T} i(t) e^{-j \omega t} d t
$$




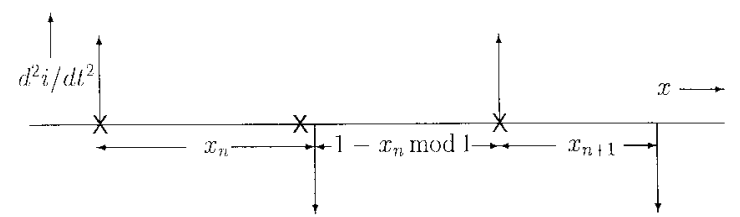

Fig. 4. The second derivative of the current waveform as a function of dimensionless time $x=t / T_{c}$. The vertical arrows represent $\delta$-functions of area $\pm \bar{V}_{O} / L$ and the clock pulses are represented by crosses.

at $\omega=\omega_{c}$, where $\omega_{c}=2 \pi / T_{c}$ is the clock angular frequency. Define $A_{m}=A\left(m \omega_{c}\right)$ for arbitrary integer $m$. Then

$$
p(t)=\sum_{m=-\infty}^{\infty} A_{m} e^{j m \omega_{c} t} .
$$

Under the assumptions 1) $R_{c}(\tau)$ is Lebesgue integrable (and therefore possesses a Fourier transform) and 2) $A(\omega)=0$ for all $\omega \neq m \omega_{c}$, integer $m$, we deduce that

$$
\begin{aligned}
R_{i}(\tau)= & R_{p+c}(\tau)=R_{p}(\tau)+R_{c}(\tau) \\
& +\lim _{T \rightarrow \infty} \frac{1}{2 T} \int_{-T}^{T} c(t)[p(t+\tau)+p(t-\tau)] d t .
\end{aligned}
$$

By considering each of the components of $p(t)$ in turn, and using assumption 2), this reduces to

$$
R_{i}(\tau)=R_{c}(\tau)+R_{p}(\tau)
$$

Hence, the PDS is

$$
\begin{aligned}
\tilde{I}(\omega) & =\int_{-\infty}^{\infty} R_{c}(\tau) e^{-j \omega \tau} d \tau+\int_{-\infty}^{\infty} R_{p}(\tau) e^{-j \omega \tau} d \tau \\
& =\tilde{I}_{c}(\omega)+\sum_{m=-\infty}^{\infty} \tilde{A}_{m} \delta\left(\omega-m \omega_{c}\right)
\end{aligned}
$$

where, by [8, ths. 11.9 and 11.10$]$ we have

$$
\tilde{A}_{m}=\left|A_{m}\right|^{2} \text {. }
$$

Our assumptions lead us to conclude that $\tilde{I}(\omega)$ is obtained by the addition of two functions, namely, a sum of $\delta$ functions at harmonics of the clock frequency and a continuous function $\tilde{I}_{c}(\omega)$ that corresponds to broadband noise. Specifically, we claim that there are no $\delta$ function peaks except those at the harmonics of the clock frequency.

Under assumption 1) of Section II, $d^{2} i / d t^{2}$ is a sequence of Dirac $\delta$ functions of alternating sign and area $\bar{V}_{O} / L$. Using the dimensionless time $x=t / T_{c}$, the situation is then as illustrated in Fig. 4.

The integration property of Fourier transforms

$$
g(t) \rightleftharpoons G(\omega) \Rightarrow \int_{-\infty}^{t} g(u) d u \rightleftharpoons \frac{1}{j \omega} G(\omega)
$$

also applies to the transformation in (11), provided $\lim _{t \rightarrow \pm \infty} i(t)$ is finite. Using the time shift property of Fourier transforms, $g(t) \rightleftharpoons G(\omega) \Rightarrow g(t-\tau) \rightleftharpoons e^{-j \omega \tau} G(\omega)$, and the fact that $\delta(t) \rightleftharpoons 1$, (11) becomes

$$
\begin{aligned}
A(\omega)= & -\frac{\bar{V}_{O}}{\omega^{2} L} \lim _{N \rightarrow \infty} \frac{1}{T_{N}}\left[\left\{1-\exp \left(-j \omega T_{c} x_{1}\right)\right\}\right. \\
& +\exp \left(-j \omega T_{c}\left[1+\left\lfloor x_{1}\right\rfloor\right]\right)\left\{1-\exp \left(-j \omega T_{c} x_{2}\right)\right\}+\cdots \\
& +\exp \left(-j \omega T_{c}\left[N-1+\left\lfloor x_{1}\right\rfloor+\cdots\right.\right. \\
& \left.\left.\left.+\left\lfloor x_{N-1}\right\rfloor\right]\right)\left\{1-\exp \left(-j \omega T_{c} x_{N}\right)\right\}\right]
\end{aligned}
$$

where $\lfloor x\rfloor$ is the integer part of $x$; the factor $-1 / \omega^{2}$ represents integrating twice; and the factor $\bar{V}_{O} / L$, which is the difference between $d i / d t$ when the switch is closed and, when it is open, sets the vertical scale correctly. The time $T_{N}$ is the total time taken for $N$ on-and-off switchings of $S$ and is therefore defined as

$$
T_{N}=T_{c} \sum_{n=1}^{N} 1+\left\lfloor x_{n}\right\rfloor .
$$

Defining the integers $J_{n}$ as

$$
J_{n}= \begin{cases}0, & n=1 \\ \sum_{k=1}^{n-1} 1+\left\lfloor x_{k}\right\rfloor=n-1+\sum_{k=1}^{n-1}\left\lfloor x_{k}\right\rfloor, & n>1\end{cases}
$$

equation (13) can be rewritten

$$
A(\omega)=-\frac{\bar{V}_{O}}{\omega^{2} L} \lim _{N \rightarrow \infty} \frac{1}{T_{N}} \sum_{n=1}^{N} e^{-j \omega T_{c} J_{n}}\left\{1-e^{-j \omega T_{c} x_{n}}\right\} .
$$

Brute force calculations of the PDS can be carried out by finding the squared modulus of the sum of this series for large $N$. The expression simplifies when $\omega=m \omega_{c}$, corresponding to the clock frequency or its harmonics. In this case, (14) becomes

$$
A_{m}=-\frac{\bar{V}_{O}}{m^{2} \omega_{c}^{2} L} \lim _{N \rightarrow \infty} \frac{1}{T_{N}} \sum_{n=1}^{N} 1-e^{-2 j \pi m x_{n}} .
$$

There are now two remaining problems: 1) calculation of the asymptotic behavior of $T_{N}$ and 2) evaluation of the sum in (15). Both of these can be addressed by using the ergodicity of the invariant density $\rho$, of the mapping $F$, in conjunction with Birkhoff's Ergodic Theorem [9]. Given an expanding mapping $F$ which preserves the ergodic measure with density $\rho(x)$ on $[0, \alpha)$

$$
\lim _{N \rightarrow \infty} \frac{1}{N} \sum_{n=1}^{N} \phi\left(F^{[n-1]}(x)\right)=\int_{0}^{\alpha} \phi(y) \rho(y) d y
$$

for (Lebesgue) almost all initial conditions $x$ and for any integrable function $\phi$ which maps $[0, \alpha)$ to $\mathcal{R}$. Here, $F^{[i]}(x)$ is the $i$ th iterate of $F$.

In order to apply the theorem we therefore need to calculate the invariant density of $F, \rho(x)$. This is the normalized density of iterates $\left\{x_{0}, x_{1}, \ldots\right\}$ of the mapping over the interval $[0, \alpha)$ and its calculation is described in the Appendix.

The asymptotic behavior of $T_{N}$ is such that

$$
\lim _{N \rightarrow \infty} \frac{T_{N}}{N}=\left\langle T_{0}\right\rangle=T_{c}(1+\langle\lfloor x\rfloor\rangle)
$$

and $\left\langle T_{0}\right\rangle$, the mean time between successive off-on transitions of $S$, can be calculated from (16)

$$
\frac{\left\langle T_{0}\right\rangle}{T_{c}}=1+\int_{0}^{\alpha}\lfloor x\rfloor \rho(x) d x .
$$

Since $T_{N} \sim N\left\langle T_{0}\right\rangle$, we can evaluate the sum in (15) in the same way

$$
\begin{aligned}
A_{m} & =-\frac{\bar{V}_{O}}{m^{2} \omega_{c}^{2} L\left\langle T_{0}\right\rangle} \int_{0}^{\alpha}\left(1-e^{-2 j \pi m x}\right) \rho(x) d x \\
& =\frac{\bar{V}_{O}}{m^{2} \omega_{c}^{2} L\left\langle T_{0}\right\rangle}\left[\int_{0}^{\alpha} e^{-2 j \pi m x} \rho(x) d x-1\right]
\end{aligned}
$$




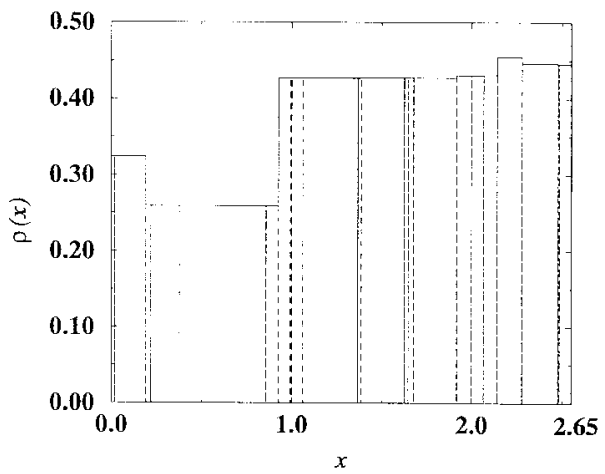

Fig. 5. The invariant density for $\alpha=2.65$. The dotted lines show the partitions.

where the second equality follows from the fact that $\rho(x)$ is normalized. Hence

$$
\begin{aligned}
\tilde{A}_{m}= & \left|A_{m}\right|^{2} \\
= & {\left[\frac{\bar{V}_{O}}{m^{2} \omega_{c}^{2} L\left\langle T_{0}\right\rangle}\right]^{2}\left[\left(\int_{0}^{\alpha} \rho(x) \cos 2 \pi m x d x-1\right)^{2}\right.} \\
& \left.+\left(\int_{0}^{\alpha} \rho(x) \sin 2 \pi m x d x\right)^{2}\right]
\end{aligned}
$$

which allows us to calculate the PDS at the $m$ th harmonic of the clock frequency.

It is now interesting to compare chaotic and periodic operation of the boost converter under the same conditions, i.e., with the same $\bar{V}_{O}$. Experimentally, this can be achieved by removing the feedback loop in Fig. 1 and, instead, driving the switch periodically, using a pulse generator with the mark-tospace ratio $(\bar{D})$ set so that the mean output voltage is the same as in the chaotic case. Starting from (15), this is equivalent to setting $x_{n}=\bar{D}, \forall n$, and $T_{N}=N T_{c}$. Hence,

$$
A_{m}=-\frac{\bar{V}_{O}}{m^{2} \omega_{c}^{2} L} \lim _{N \rightarrow \infty} \frac{1}{N T c} \sum_{n=1}^{N} 1-e^{-2 j \pi m \bar{D}} .
$$

Eliminating $\overline{\Delta i}$ between (9) gives $\bar{D}=\alpha /(1+\alpha)$ and so the PDS for periodic operation is

$$
\tilde{A}_{m}=\left|A_{m}\right|^{2}=2\left[\frac{\bar{V}_{O}}{m^{2} \omega_{c}^{2} L T_{c}}\right]^{2}\left(1-\cos \frac{2 \pi m \alpha}{1+\alpha}\right) .
$$

\section{RESULTS}

The approximate $\rho(x)$, using twenty iterations of the scheme described in the Appendix, for $\alpha=2.65$, is shown in Fig. 5.

Equation (17) can now be used to calculate $\left\langle T_{0}\right\rangle$ as a function of $\alpha$. A plot of $\left\langle T_{0}\right\rangle / T_{c}$ against $\alpha$ is shown in Fig. 6. Note that $\left\langle T_{0}\right\rangle=2.00538 T_{c}$ at $\alpha=2.65$.

Using the invariant density and (18), we can calculate the PDS as explained in the previous section. It is also possible, although slower, to estimate this by brute force (14) and, for the purposes of comparison, this was also carried out. In Fig. 7, these two calculations are compared with each other and also

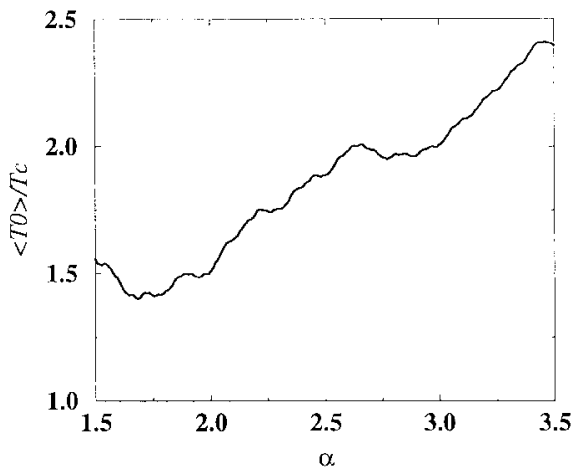

Fig. 6. The variation of $\left\langle T_{0}\right\rangle / T_{c}$ with $\alpha$. This was calculated from (17), using $\rho(x)$ approximated by 50 iterations of the scheme outlined in the Appendix.

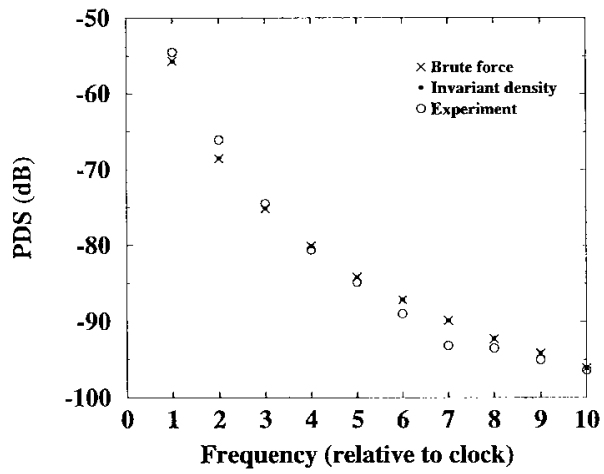

Fig. 7. Three-way comparison of the inductor current PDS at the clock frequency and its harmonics, by brute force [(14)], the invariant density method, and experiment. The value of $\alpha$ is 2.65 .

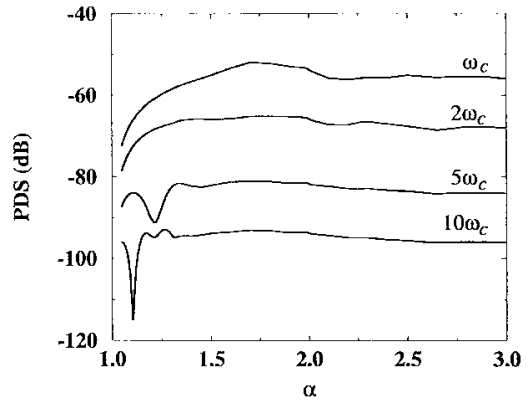

Fig. 8. The PDS at $\omega_{c}, 2 \omega_{c}, 5 \omega_{c}$, and $10 \omega_{c}$ as a function of $\alpha$, calculated from the invariant density.

with the results from an experimental $i(t)$ waveform. ${ }^{1}$ The experimental details are given in the next section.

It is interesting to note how the PDS at a given harmonic of the clock frequency varies with $\alpha$, and this is displayed in Fig. 8 for the fundamental and the second, fifth, and tenth harmonics. For a comparison of chaotic with periodic operation, see Fig. 9. This shows how the calculated spectral peaks at $\omega_{c}, 2 \omega_{c}, 5 \omega_{c}$, and $10 \omega_{c}$ vary with $\alpha$ when the converter is allowed to operate chaotically and when it is forced to operate periodically, $\bar{V}_{O}$ being the same in each case. For the fundamental and the second harmonic, the PDS is

\footnotetext{
${ }^{1}$ The vertical scale on all plots whose vertical axis is labeled PDS (dB) is $10 \log _{10} \tilde{A}_{m}$
} 


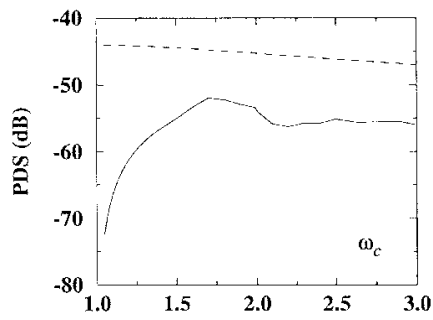

(a)

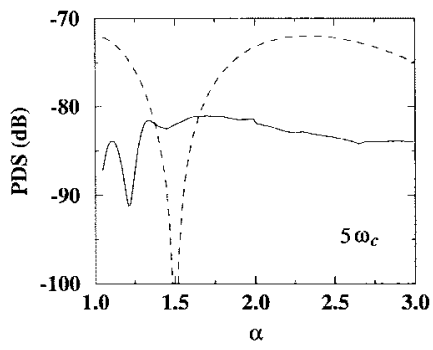

(c)

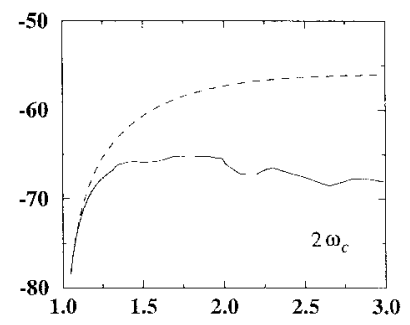

(b)

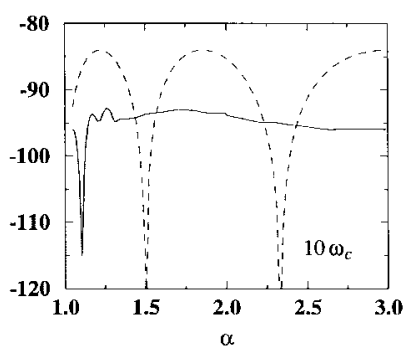

(d)
Fig. 9. A comparison of chaotic and periodic operation. (a) The PDS at $\omega_{c}$, (b) $2 \omega_{c}$, (c) $5 \omega_{c}$, (d) and $10 \omega_{c}$ is shown as a function of $\alpha$. Chaotic: continuous line, periodic operation with the same $\bar{V}_{O}$ : dashed line.

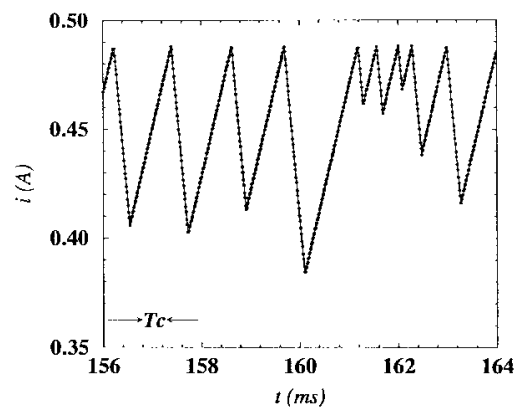

Fig. 10. Experimental chaotic current waveform.

always less in the chaotic case. This is usually but not always so for the other harmonics considered.

\section{EXPERIMENTAL RESULTS}

The peak-current controlled boost converter shown in Fig. 1 was built using $R=293 \Omega, L=104 \mathrm{mH}, C=220 \mu \mathrm{F}$, $V_{I}=10.45 \mathrm{~V}, \bar{V}_{O}=32.3 \mathrm{~V}$, reference current $I_{\text {ref }}=0.5 \mathrm{~A}$, and clock period $T_{c}=400 \mu \mathrm{s}$. The total inductor series resistance $r_{L}=(1.0+2.33) \Omega$, the $1 \Omega$ being a current sensing resistor. With these parameter values the circuit behaved chaotically. The output voltage had a chaotic ripple of peak-topeak amplitude $\approx 0.6 \mathrm{~V}$. Assumption 1 ) of Section II requires that $C R / T_{c} \gg 1$, which is satisfied by our values for which $C R / T_{c} \approx 160$.

An analogue-to-digital converter was used to monitor the inductor current $i(t)$. A section of the experimental current waveform, sampled at $40 \mathrm{kHz}$, is shown in Fig. 10. An experimental version of the mapping given in (6) was reconstructed from the turning points of a rather longer portion of this waveform and is shown in Fig. 11. The shape is exactly that expected from the theoretical mapping, (6), confirming that the 1-D approximation is appropriate.

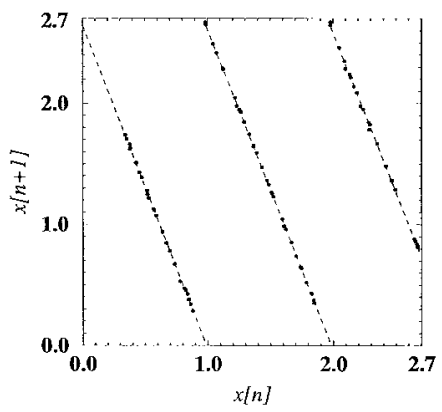

Fig. 11. Experimental mapping deduced from the measured waveform; cf. (6). The dotted lines are a least squares fit to each of the linear portions of the mapping.

The value of $\alpha$ that applies to this converter can be estimated in several ways. These include the following.

1. By definition, $\alpha=$ Effective $\left(\bar{V}_{O} / V_{I}\right)-1$. The actual value of $V_{I}$ was $10.45 \pm 0.06 \mathrm{~V}$. The mean voltage drop across $r_{L}$ was measured as $1.4 \pm 0.06 \mathrm{~V}$ and, hence, the effective $V_{I}=9.05 \pm 0.12 \mathrm{~V}$. The measured value of $\bar{V}_{O}$ was $32.3 \pm 0.26 \mathrm{~V}$ and the drop across the diode was $0.8 \pm 0.1 \mathrm{~V}$, hence, the effective $\bar{V}_{O}=33.1 \pm 0.36 \mathrm{~V}$. These results give $\alpha=2.66 \pm 0.09$.

2. The gradients, with standard errors, of the three negative slope portions of the experimental mapping in Fig. 11 were found by least squares fit, giving a second experimental estimate of $\alpha=2.66 \pm 0.02$.

3. Equation (10), derived from an approximate model of the circuit neglecting parasitic resistances, has the three roots $\bar{V}_{O}=-35.4,-0.32$, and $35.7 \mathrm{~V}$, the first two of which can be rejected since they are negative. This gives $\alpha=2.94$.

Finally, the discrete-time analogue of (14) was calculated for a time series consisting of $K=2^{16}$ experimental samples of $i(t)$ according to

$$
I_{n}=\frac{1}{K} \sum_{k=0}^{K-1} i_{k} e^{2 \pi j k n / K}
$$

where $i_{k}=i(k h)$, with $h$ the sampling interval. The number of switch transitions that occur in the time of $K=2^{16}$ samples is $N=1921$ so, for a direct comparison, a brute force calculation (14) was also carried out for this $N$. The results are displayed in Fig. 12 and it can be seen that there is a good measure of agreement between $\left|I_{n}\right|^{2}$ and the brute-force calculation.

\section{DISCUSSION}

The simplified 1-D mapping is adequate for practical versions of a chaotically operating boost converter. As published in [11] for a different nonlinear circuit, the invariant density of this mapping can be derived. From this, the power density spectrum of the input current can be calculated, confirming an assertion we made in [12], that "the parameters at which subharmonics and chaos commence can be predicted accurately, as can ... the frequencies emitted from the power supply." Measurements verify the accuracy of our calculations, which 


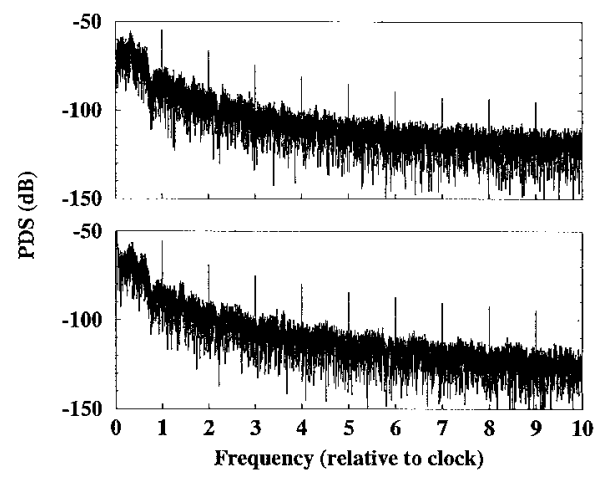

Fig. 12. Experimental measurement (top) and brute force calculation (bottom) of the PDS of $i(t)$ for $\alpha=2.65$.

may therefore be used with confidence as a design tool in the specification of boost converter circuits where the EMC targets are tightly controlled. One important application is in high power-factor single-phase rectifiers for mains power supplies, e.g. [13].

A recent publication [14] sets out the conditions for the existence of robust chaos, which is characterized by the absence of periodic windows and coexisting attractors in some region of parameter space. Note that the mapping $F$ has a single chaotic attractor for $\alpha>1$ and, hence, the boost converter described here is robustly chaotic.

Although the full circuit description results in a 2-D mapping, we believe that the practical benefits of extending the analysis to this case are minimal, particularly in view of the extra theoretical and computational difficulties this would raise. There could, however, be an advantage to examining the sensitivity of the spectrum to the nearly 1-D approximation.

Another extension of this work would involve calculating the continuous part of the PDS away from the clock frequency and its harmonics $\tilde{I}_{c}(\omega)$.

Our results may, of course, be applicable to other physical systems that can be described by a piecewise-linear 1-D mapping.

\section{CONCLUSION}

We have shown that spectral peaks of the input current in a boost converter in chaotic operation can be calculated. The predictions are in satisfactory agreement with measurements on a practical circuit. The calculation method may, therefore, be adopted by engineers for design purposes.

\section{APPENDIX}

The Perron-Frobenius operator $P$ related to the mapping $F$ (6) is defined by

$$
P(g)(x)=\sum_{y=F^{-1}(x)} \frac{1}{\left|F^{\prime}(y)\right|} g(y) .
$$

For the expanding map $F$, its invariant density $\rho(x)$ can be calculated as the $\operatorname{limit}_{n \rightarrow \infty} \rho_{n}(x)$ where $\rho_{n}(x)=$ $P\left(\rho_{n-1}\right)(x)$, with $\rho_{0}(x)$ being any nonnegative function with

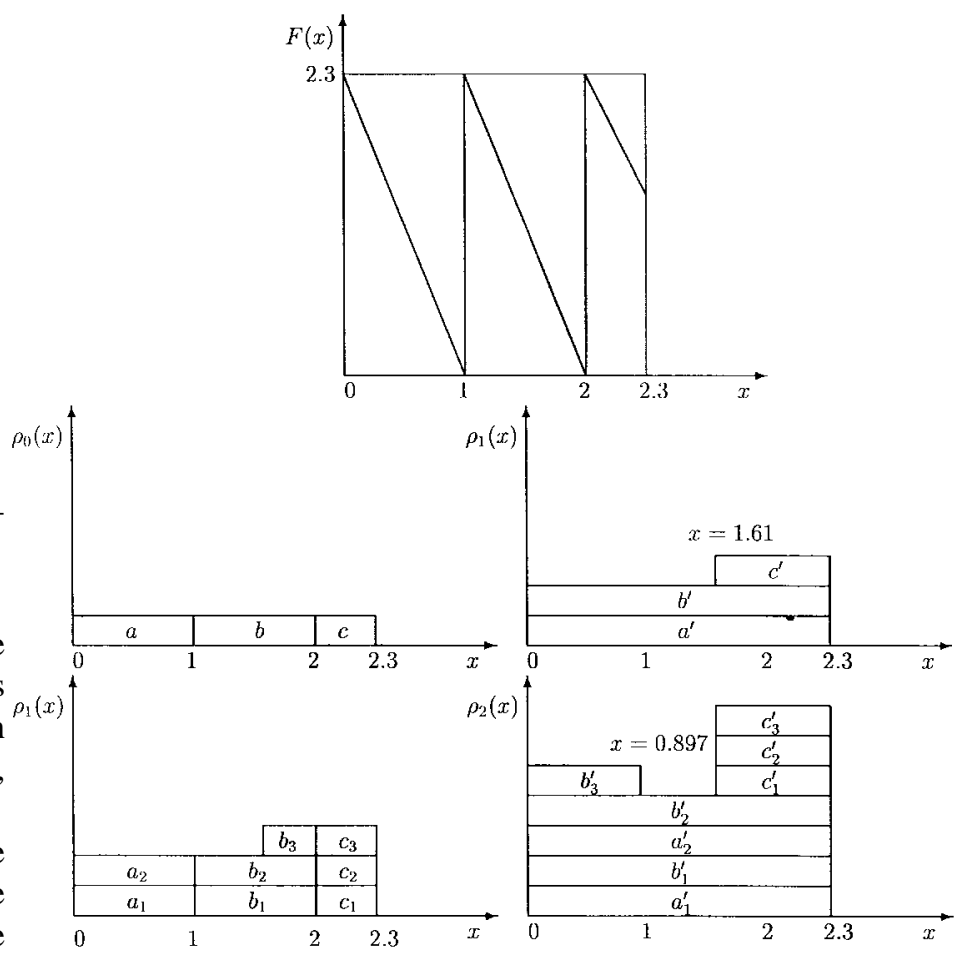

Fig. 13. The mapping $F(x)$ and the algorithm for calculating the invariant density $\rho(x)$ by successive approximations $\rho_{0}, \rho_{1}, \rho_{2} \ldots$, with $\alpha=2.3$.

integral one (e.g., a constant). This is illustrated in Fig. 13, in which $\alpha=2.3$, but the scheme converges for any $\alpha>1$.

The obvious choice for $\rho_{0}(x)$ is therefore

$$
\rho_{0}(x)= \begin{cases}\frac{1}{\alpha}, & 0 \leq x<\alpha \\ 0, & \text { otherwise }\end{cases}
$$

which is clearly normalized. The $x$ axis is split into three intervals, labeled $a,[0,1) ; b,[1,2)$; and $c,[2, \alpha]$. The densities on $a$ and $b$ are mapped by $F$ uniformly and with equal weighting ${ }^{2}$ onto $a^{\prime}$ and $b^{\prime}$, where $a^{\prime}=b^{\prime}=[0, \alpha]$, but the density on $c$ is mapped onto $c^{\prime}=[\alpha, F(\alpha)]=[2.3,1.61]$. The first approximation to $\rho, \rho_{1}(x)$ then comprises the sum of these three densities. To calculate $\rho_{2}$ from $\rho_{1}$, the same procedure is used, except that this time there are eight subintervals to be considered, $a_{1}, a_{2}, b_{1} \ldots b_{3}$, and $c_{1} \ldots c_{3}$. All the intervals except $b_{3}=[1.61,2)$ have been treated before and are dealt with in the same way again; $b_{3}$ is mapped to $b_{3}^{\prime}=$ $\left[0, F^{[2]}(\alpha)\right]=[0,0.897]$. The sum of these eight densities gives $\rho_{2}$.

A computer program was written to iterate the Perron-Frobenius operator $n$ times and plot the result.

Note that this algorithm does not require the mapping $F(x)$ to have a finite number of Markov partitions [10]. It can also be modified to calculate the invariant densities of any piecewiselinear mapping, provided that the densities on each interval are mapped with a weighting factor equal to the modulus of the reciprocal of the gradient of the mapping in each interval.

\footnotetext{
${ }^{2}$ This is because $|d F / d x|=\alpha$ everywhere except at $x=1,2, \ldots$.
} 


\section{REFERENCES}

[1] J. H. B. Deane and D. C. Hamill, "Improvement of power supply EMC by chaos," Electron. Lett., vol. 32, no. 12, p. 1045, June 1996.

[2] J. H. B. Deane, "Chaos in a current-mode controlled boost dc-dc converter," IEEE Trans. Circuits Syst., vol. 39, pp. 680-683, Aug. 1992.

[3] D. C. Hamill, J. H. B. Deane, and D. J. Jefferies, "Modeling of chaotic DC-DC converters by iterated nonlinear mappings," IEEE Trans. Power Electron., vol. 7, pp. 25-36, Jan. 1992.

[4] J. H. B. Deane and D. C. Hamill, "Instability, subharmonics and chaos in power electronic systems," IEEE Trans. Power Electron., vol. 5, pp. 260-268, Mar. 1990.

[5] S. Banerjee and K. Chakrabarty, "Nonlinear modeling and bifurcations in the boost converter," IEEE Trans. Power Electron., vol. 13, pp. 252-260, Mar. 1998.

[6] A. Rényi, "Representation for real numbers and their ergodic properties," Acta Math. Acad. Sci. Hung., vol. 8, pp. 477-493, 1957.

[7] A. Lasota and M. Mackey, "Chaos, fractals and noise: Stochastic aspects of dynamics" (Applied Mathematical Sciences Series). New York: Springer-Verlag, 1994, vol. 9.

[8] D. C. Champeney, A Handbook of Fourier Theorems. Cambridge, U.K.: Cambridge Univ. Press, 1987.

[9] J.-P. Eckmann and D. Ruelle, "Ergodic theory of chaos and strange attractors," Rev. Modern Phys., vol. 57, no. 3, pt. 1, pp. 617-656, 1985

[10] S. H. Isabelle, "A signal processing framework for the analysis and application of chaotic systems," Ph.D. dissertation, Massachusetts Inst. Technol., Cambridge, MA, 1995.

[11] T. Saito, "A chaos generator based on a quasiharmonic oscillator," IEEE Trans. Circuits Syst., vol. 32, pp. 320-331, Apr. 1985.

[12] D. C. Hamill and D. J. Jefferies, "Subharmonics and chaos in a controlled switched-mode power converter," IEEE Tran. Circuits Syst., vol. 35, pp. 1059-1061, Aug. 1988.

[13] L. H. Dixon, "High power factor preregulator for off-line power supplies," in Unitrode Switching Regulated Supply Design Seminar Manual (SEM-700), Unitrode Corp., sec. I2, 1990.

[14] S. Banerjee, J. A. Yorke, and C. Grebogi, "Robust chaos," Phys. Rev. Lett., vol. 80, pp. 3049-3052, 1998

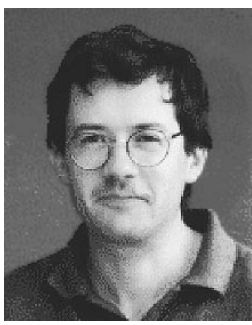

Jonathan H. B. Deane received the B.A. degree in physics from Oxford University, Oxford, U.K., in 1982.

After teaching and working in the European Patent Office, the Hague, the Netherlands, he became a Research Fellow in the Department of Electrical and Electronic Engineering, the University of Surrey, Guildford, U.K., in 1986. He completed his Ph.D. dissertation on the subject of chaos in electronic circuits in 1990. His research interests include nonlinear dynamics of electronic systems, chaos in power electronics and modeling of nonlinear circuits.

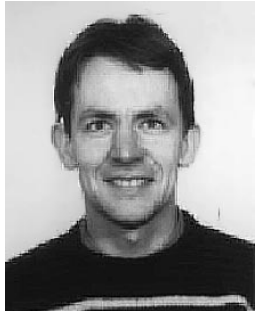

Peter Ashwin received the B.A. and C.A.S. degrees in mathematics from Cambridge University, Cambridge, U.K., in 1987 and 1988, respectively, and the Ph.D. degree in mathematics from Warwick University, U.K., in 1991.

He is a Lecturer at the Department of Mathematics and Statistics, the University of Surre, Guildford, U.K. His current research interests are in the areas of theory, applications and experiments involving nonlinear dynamics, especially coupled dynamical systems, dynamics with discontinuities, and the influence of noise on deterministic dynamics.

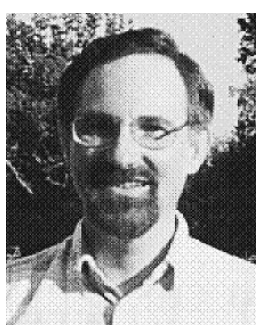

David C. Hamill (SM'94) was born in London, U.K. He received the B.Sc. degree in engineering and the M.Sc. degree from the University of Southampton, Southampton, U.K., and the Ph.D. degree from the University of Surrey, Guildford, U.K

After practicing as a design engineer and a consultant, he was technical director of PAG Ltd., London, U.K., for seven years. In 1986 he joined the University of Surrey, where he is currently a Senior Lecturer in the Surrey Space Centre. His research interests include dc-dc conversion, space power systems and nonlinear dynamics.

Dr. Hamill was an Associate Editor of the IEEE TRAnsactions on Power ELECTRONICS from 1994 to 1996 . He is a member of the IEE and a Chartered Engineer.

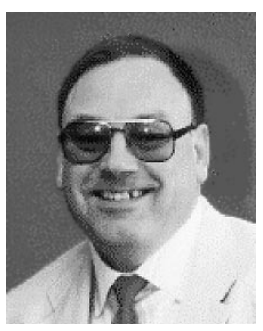

David J. Jefferies received the B.A. degree in physics from Oxford University, Oxford, U.K., in 1965 and the M.S. and Ph.D. degrees in applied physics from Stanford University, Palo Alto, CA, in 1968 and 1979.

He has held research posts at Stanford, Aberdeen, and Nottingham Universities and from 1979 to 1983 was in charge of teaching the electronics content of the undergraduate physics course at Oxford. Since 1983 he has been at the University of Surrey, Guildford, U.K., doing research in chaotic electronic processes and teaching undergraduate and graduate courses. He has held numerous research grants in the experimental study of chaotic electronic systems.

Dr. Jefferies was the Editor of the International Journal of Electronics from 1988 to $1996 . \mathrm{He}$ is a member of Sigma Xi. 